\title{
Efficient on-chip source of microwave photon pairs in superconducting circuit QED
}

\author{
Florian Marquardt \\ Physics Department, Center for NanoScience, and Arnold Sommerfeld Center for Theoretical Physics, \\ Ludwig-Maximilians Universität Munich, Theresienstrasse 37, 80333 Munich, Germany
}

(Received 10 August 2007; published 13 November 2007)

\begin{abstract}
We describe a scheme for the efficient generation of microwave photon pairs by parametric down-conversion in a superconducting transmission line resonator coupled to a Cooper-pair box serving as an artificial atom. By properly tuning the first three levels with respect to the cavity modes, the down-conversion probability may reach the percentage level at good fidelity. We show this by numerically simulating the dissipative quantum dynamics of the coupled cavity-box system and discussing the effects of dephasing and relaxation in the solid state environment. The setup analyzed here might form the basis for a future on-chip source of entangled microwave photons, e.g., using Franson's idea of energy-time entanglement.
\end{abstract}

DOI: 10.1103/PhysRevB.76.205416

PACS number(s): 03.67.Mn, 42.50.Dv, 42.50.Pq, 85.25.-j

\section{INTRODUCTION}

The generation of photon pairs by parametric downconversion $(\mathrm{PDC})^{1-5}$ represents one of the basic ways to create nonclassical states of the electromagnetic field, which has found numerous applications so far. The conditional detection of one of the photons enables the production of single photon Fock states. ${ }^{2,6}$ Furthermore, PDC is the primary method to generate entangled pairs of particles. Apart from the possibility of testing Bell inequalities, ${ }^{3-7}$ this represents a crucial ingredient for a multitude of applications in the field of quantum information science, ranging from quantum teleportation through quantum dense coding to quantum key distribution. ${ }^{8}$

With the advent of superconducting circuit quantum electrodynamics, ${ }^{9,10}$ it will now be possible to take over many of the concepts that have been successful in the field of quantum/atom optics and to transfer them to the domain of microwave photons guided along coplanar waveguides on a chip, interacting with superconducting qubits. ${ }^{11-14}$ Recent experiments have realized the strong-coupling limit of the Jaynes-Cummings model known in atom optics, employing a superconducting qubit as an artificial two-level atom and coupling it resonantly to a harmonic oscillator (i.e., a cavity mode $^{10}$ or a superconducting quantum interference device ${ }^{15}$ ). Dispersive quantum non-demolition (QND) measurements of the qubit state, Rabi oscillations, and Ramsey fringes have been demonstrated, ${ }^{16,17}$ leading to a fairly detailed quantitative understanding of the system, which behaves almost ideally as predicted by theory. ${ }^{9}$ Based on these successes, new approaches to quantum computing have been proposed, such as a two-dimensional grid of microwave resonators with qubits at the intersections ${ }^{18}$ (termed a "cavity grid").

In this paper, we will analyze a scheme that implements parametric down-conversion of microwave photons entering a transmission line resonator coupled to a Cooper pair box (CPB) providing the required nonlinearity (Fig. 1). This represents the limit of a single artificial atom taking the place of the nonlinear crystal usually employed in optical PDC experiments, ${ }^{1-3,5}$ with the cavity enhancing the PDC rate (see Ref. 19). In contrast to other solid state PDC proposals, ${ }^{20-22}$ both the basic cavity setup and the possibility of ejecting the generated photons into single-mode transmission lines with a high degree of reliability are already an experimentally proven reality. ${ }^{10,16,17}$ Recently, squeezing and degenerate parametric down-conversion have been analyzed theoretically $^{23}$ for a circuit QED setup coupling a charge qubit to two cavity modes. However, unlike the experiments and most of the theoretical investigations mentioned above, in this paper we propose to go beyond the regime where the box may be regarded as a two-level system (qubit), making use of its first three levels. By further employing its advantage over real atoms, namely, its tunability via the applied magnetic flux and the gate voltage, this enables us to bring the transitions between the first three box levels into (near) resonance with the first three cavity modes (Fig. 2), thereby drastically enhancing the resulting probability of (nondegenerate) parametric down-conversion $|3 \omega\rangle \mapsto|\omega\rangle \otimes|2 \omega\rangle$. This represents the major advantage of the present scheme. We treat the full quantum-dissipative dynamics of the box-cavity system, incorporating the radiation of photons from the cavity as well as nonradiative decay processes and dephasing in the CPB. We will present results for the down-conversion efficiency, discuss the minimization of unwanted loss processes, and comment on possible applications in the end.

\section{MODEL}

The CPB is a device ${ }^{14}$ in which Cooper pairs can tunnel between two superconducting islands due to a Josephson

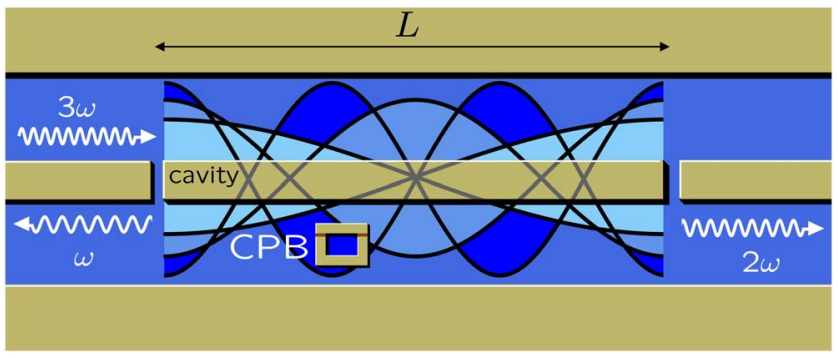

FIG. 1. (Color online) Schematic setup for the proposed parametric down-conversion (PDC) experiment in superconducting circuit cavity electrodynamics, with a Cooper-pair box (CPB) interacting with the three lowest modes of a transmission line resonator, whose voltage distributions are shown. 


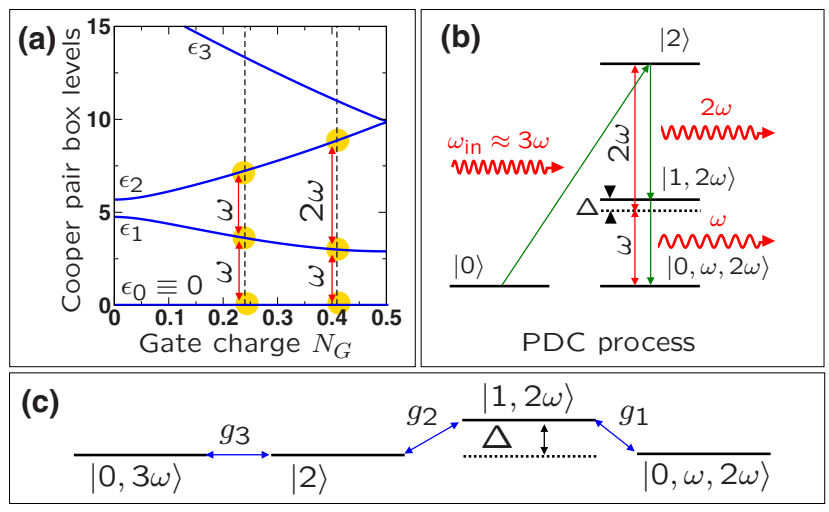

FIG. 2. (Color online) (a) Excitation energies (transition frequencies) of the $\mathrm{CPB}$, measured with respect to the ground state energy $\epsilon_{0} \equiv 0$ as a function of gate charge $N_{G}$. The energies are plotted in units of $E_{C}$ for $E_{J} / E_{C}=3$. At particular gate values (away from the "qubit" regime near $N_{G}=1 / 2$ ), the CPB transition frequencies are related in an integer ratio, $\left(\epsilon_{2}-\epsilon_{1}\right) / \epsilon_{1}=1: 1$ or $2: 1$, respectively. This enables us to match them with the cavity modes, giving rise to particularly efficient degenerate or nondegenerate PDC ( $2 \omega \mapsto \omega+\omega$ or $3 \omega \mapsto \omega+2 \omega$, respectively). (b) Simplified transition scheme for the nondegenerate PDC process considered in the text, with a detuning $\Delta$ of the intermediate state $|1,2 \omega\rangle: \epsilon_{1}=\omega+\Delta$. In this plot, only the energy contribution of the CPB is indicated in terms of the vertical position of the levels. The incoming and outgoing photons are displayed as well. (c) Alternative illustration of the same scheme: Here, the level position indicates the full energy of the $\mathrm{CPB}+$ cavity system, and the coupling strengths governing the individual transitions are shown (see main text).

coupling $E_{J}$ (tunable by an external magnetic flux in a splitjunction geometry). The number of transferred Cooper pairs $\hat{N}$ determines the charging energy, whose scale $E_{C}=e^{2} / 2 C_{\Sigma}$ is set by the total capacitance $C_{\Sigma}$ of the box island and which can be controlled by the application of an external gate voltage (expressed in terms of a gate charge $N_{G}$ ),

$$
\hat{H}_{\mathrm{CPB}}=4 E_{C}\left(\hat{N}-N_{G}\right)^{2}-\frac{E_{J}}{2} \sum_{N}|N+1\rangle_{c}\left\langle\left. N\right|_{c}+\right.\text { H.c. }
$$

Here, $|N\rangle_{c}$ represents a charge state of the CPB. Two gaps in a superconducting coplanar waveguide act as mirrors of a cavity for microwave photons, $\hat{H}_{\text {cavity }}=\sum_{j=1}^{3} \omega_{j} \hat{a}_{j}^{\dagger} \hat{a}_{j}$, where we will focus our attention on the three lowest-lying cavity modes with $\omega_{j}=j \omega$ (we set $\hbar \equiv 1$ ). The electric field inside the cavity acts on the $\mathrm{CPB},{ }^{9}$ adding a quantum-mechanical component to the gate charge $N_{G}$ and leading to an interaction $\hat{H}_{\text {int }}=\left[\Sigma_{j=1}^{3} g_{j}\left(\hat{a}_{j}+\hat{a}_{j}^{\dagger}\right)\right] \hat{N}$. The coupling constants $g_{j}$ $=g_{0} \sqrt{j} \phi_{j}(x)$, with $g_{0}=2 \frac{e C_{g}}{C_{\Sigma}} \sqrt{\omega / L c}$ and $C_{g}$ the gate capacitance, are given in terms of the mode functions $\phi_{1}(x)$ $=\sin (x \pi / L), \quad \phi_{2}(x)=\cos (2 \pi x / L), \quad$ and $\quad \phi_{3}(x)=\sin (3 \pi x / L)$ (Fig. 1), which are defined on the interval $x=-L / 2 \cdots L / 2$.

The full Hamiltonian forming the basis of our analysis is thus given by

$$
\hat{H}=\hat{H}_{\text {cavity }}+\hat{H}_{\mathrm{CPB}}+\hat{H}_{\text {int }}+\hat{H}_{\text {env }}=\hat{H}_{0}+\hat{H}_{\text {env }},
$$

where $\hat{H}_{\text {env }}$ includes the coupling to the environment, which describes microwave photons leaking out of the cavity, as well as relaxation and decoherence processes acting on the CPB.

\section{BASIC CONSIDERATIONS}

Three basic features distinguish such a setup from the usual PDC experiments employing nonlinear crystals: (i) There is no momentum conservation, as the system is zero dimensional. This is similar to nonlinear optical processes induced in a short crystal, where momentum conservation is spoiled according to the uncertainty principle. (ii) Energy conservation is much more restrictive, as the set of possible frequencies is limited to the discrete cavity modes, resulting in a resonant enhancement of the PDC process. In contrast to a passive filtering scheme, the bandwidth of the generated photons is reduced without diminishing the signal intensity. (iii) The microwave polarization is fixed and thus cannot be used for entanglement. At the end of this paper, we will point out other options that can be explored.

\section{ESTIMATION OF THE PARAMETRIC DOWN-CONVERSION RATE}

If the CPB is operated as a two-level system (qubit), ${ }^{23}$ the decay of a $3 \omega$ photon into two lower-energy photons involves at least one intermediate virtual state having an energy detuning of the order of $\omega$, which contributes a small factor $(g / \omega)^{2}$ to the PDC rate.

We can enhance the PDC rate by exploiting at least three levels of the CPB and tuning the Josephson coupling $E_{J}$ and the gate charge $N_{G}$ to make the transitions between the first three CPB energy levels $|0\rangle,|1\rangle$, and $|2\rangle$ resonant with the cavity modes (Fig. 2). We consider the PDC process $|0,3 \omega\rangle \mapsto|2\rangle \mapsto|1,2 \omega\rangle \mapsto|0, \omega, 2 \omega\rangle$, with $\quad \epsilon_{2} \approx 3 \omega$ and $\epsilon_{1}=\omega+\Delta$. This reduces the largest energy denominator to the detuning $\Delta$, resulting in an enhancement of the PDC rate by a factor of $(\omega / \Delta)^{2}$. What limits the enhancement? If $\Delta$ is too small, the intermediate state $|1,2 \omega\rangle$ will acquire a significant population. As a result, the temporal correlation between photons would be destroyed, and nonradiative decays $|1,2 \omega\rangle \mapsto|0,2 \omega\rangle$ may occur, without emitting the second photon of frequency $\omega$. Clearly, there is a tradeoff between the achieved PDC probability and the fidelity of downconversion. This will be confirmed by the detailed simulations below.

We will find that it is possible to achieve a PDC probability in the percentage range that surpasses that of the most efficient modern optical PDC schemes ${ }^{24}$ (which have a PDC probability of about $10^{-4}$, though the absolute PDC rate in those experiments is about $10^{9}$ times larger due to the drastically higher input power). Earlier well-known optical PDC experiments ${ }^{5}$ generated less than one usable coincidence detection event for every $10^{13}$ incoming photons. 


\section{SIMULATION OF THE QUANTUM-DISSIPATIVE DYNAMICS}

In the ideal case, one could integrate out the intermediate state, yielding an effective PDC term of the form $|0\rangle\langle 2| \hat{a}_{1}^{\dagger} \hat{a}_{2}^{\dagger}+$ H.c. However, here, we have to take into account all loss processes by solving for the full dynamics of the $\mathrm{CPB} /$ cavity system under an external microwave drive of frequency $\omega_{\text {in }} \approx 3 \omega$, using a Markoff master equation of Lindblad form,

$$
\frac{d \hat{\rho}}{d t}=\left(\mathcal{L}_{0}+\mathcal{L}_{\text {drive }}+\mathcal{L}_{\text {cavity }}^{\text {decay }}+\mathcal{L}_{\mathrm{CPB}}^{\text {relax }}+\mathcal{L}_{\mathrm{CPB}}^{\text {deph }}\right) \hat{\rho} .
$$

Here, $\mathcal{L}_{0} \hat{\rho}=-i\left[\hat{H}_{0}, \hat{\rho}\right]$, and the external microwave input, at a frequency $\omega_{\text {in }} \approx 3 \omega$ and with an amplitude $\alpha$, is described by $\mathcal{L}_{\text {drive }} \hat{\rho}=-i\left[\hat{H}_{\text {drive }}(t), \hat{\rho}\right]$, with $\hat{H}_{\text {drive }}(t)=\alpha \hat{a}_{3}^{\dagger} e^{-i \omega_{\text {in }} t}+$ H.c.

The dissipative terms in the Liouvillian are of Lindblad form,

$$
\mathcal{L}[\hat{A}] \hat{\rho} \equiv \hat{A} \hat{\rho} \hat{A}^{\dagger}-\frac{1}{2} \hat{A}^{\dagger} \hat{A} \hat{\rho}-\frac{1}{2} \hat{\rho} \hat{A}^{\dagger} \hat{A} .
$$

They describe the decay of each cavity mode at a rate $\kappa_{j}$, $\mathcal{L}_{\text {cavity }}^{\text {decay }}=\Sigma_{j} \kappa_{j} \mathcal{L}\left[\hat{a}_{j}\right]$; pure dephasing processes in the CPB that do not lead to transitions between levels (at rates $\gamma_{\varphi, j}$ ), $\mathcal{L}_{\mathrm{CPB}}^{\mathrm{deph}}=\sum_{j} \gamma_{\varphi, j} \mathcal{L}[|j\rangle\langle j|]$; and nonradiative relaxation processes leading from a level $l$ to a lower-energy level $j$ of the qubit, $\mathcal{L}_{\mathrm{CPB}}^{\text {relax }}=\Sigma_{j<l} \gamma_{j \leftarrow l} \mathcal{L}[|j\rangle\langle l|]$.

We keep only resonant terms ("rotating wave approximation") in the CPB-cavity interaction and go over to a frame rotating at $\omega_{\text {in }}$ by applying the transformation $\exp (i \hat{W} t) \hat{A} \exp (-i \hat{W} t)$ to $\hat{H}_{0}+\hat{H}_{\text {drive }}$ and the density matrix. This eliminates the time dependence in $\hat{H}_{\text {drive }}$ and replaces $\hat{H}_{0}$ by $\hat{H}_{0}-\hat{W}$, with $3 \hat{W} / \omega_{\text {in }}=\sum_{j} j \hat{a}_{j}^{\dagger} \hat{a}_{j}+|1\rangle\langle 1|+3| 2\rangle\langle 2|$.

We have obtained numerical solutions of the master equation for a wide range of parameters. All these simulations have been performed in a Hilbert space that has been truncated under the assumption of a small external drive. (The maximum excitation energy of the qubit+cavity system is restricted to $3 \omega$, and the down-conversion rate is linear in the input power.)

In order to obtain a good PDC probability, we place the $\mathrm{CPB}$ at $x=0.3 L$, where the amplitudes (and, therefore, the coupling constants) of all the three relevant modes are comparable (see Fig. 1). Moreover, for the plots shown below, we have used the parameter values $g_{0} / \omega=10^{-2}, \kappa_{j} / \omega=10^{-4}$, $\gamma_{j \leftarrow l} / \omega=10^{-5}(j<l)$, and $\gamma_{\varphi, j} / \omega=2 \times 10^{-4}(j>0)$, where $\omega \sim 10 \mathrm{GHz}$ in typical experiments. ${ }^{10}$ Although these parameters (with even better dephasing rates) have been reached in recent experiments, we have to emphasize that those experiments have been conducted at the degeneracy point, where dephasing due to charge noise is strongly suppressed. In contrast, the PDC scheme developed here operates at gate charge values $N_{G}$ that are removed by 0.1 from the degeneracy point (see Fig. 2). As a consequence, the currently achievable values of the dephasing rate would still be at least by about a factor of 20 larger than those assumed here. On the other hand, further reduction of the dephasing rate will be a gen-

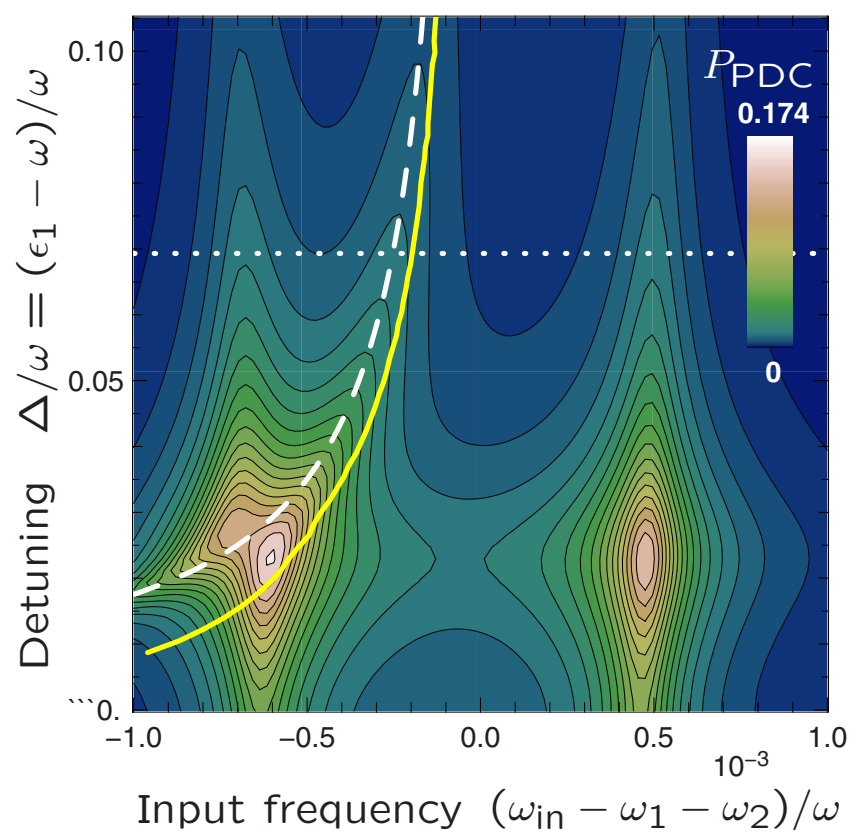

FIG. 3. (Color online) Parametric down-conversion probability $P_{\mathrm{PDC}}=P(|\omega, 2 \omega\rangle) \kappa^{2} /|\alpha|^{2}$ as a function of the microwave input frequency $\omega_{\text {in }}$ and the detuning of the intermediate state. The dashed line indicates the analytical resonance condition (see main text), the full line denotes the location of minimal $Q_{1}$ (high fidelity of PDC), and the dotted line is the cross section shown in Fig. 4.

eral prerequisite for progress in quantum computing applications, which will then facilitate the present scheme.

At any given value of $E_{J}$, one can fulfill the bare resonance condition $\epsilon_{2}=3 \omega$ by tuning to some $N_{G}=N_{G}^{*}\left[E_{J}\right]$. A small additional "offset gate charge" $\delta N_{G}$ then mainly changes the level $\epsilon_{2}$, while $E_{J}$ itself is used to tune $\epsilon_{1}$. The plots discussed below have been obtained at fixed $\delta N_{G}$ (unless noted otherwise), while changing other parameters (such as $E_{J}$ ).

\section{DISCUSSION}

In order to interpret the results, we note that the production of photon pairs at a rate $\Gamma_{\text {PDC }}$ is balanced by the decay of photons out of the cavity, at a rate $\kappa$. Thus, in an ideal lossless cavity PDC scheme, the probabilities to find the cavity in the states $|\omega, 2 \omega\rangle,|\omega\rangle$, and $|2 \omega\rangle$ all become equal to $\Gamma_{\mathrm{PDC}} /(2 \kappa)$. Therefore, we define $\Gamma_{\mathrm{PDC}} /(2 \kappa) \equiv P_{|\omega, 2 \omega\rangle}$. In combination with the rate $R$ of incoming photons, $R=2|\alpha|^{2} / \kappa$, this can be used to define the PDC probability (chance of a given photon undergoing PDC): $P_{\mathrm{PDC}}$ $=\Gamma_{\mathrm{PDC}} / R=P_{|\omega, 2 \omega\rangle} \kappa^{2} /|\alpha|^{2}$. We will use this as a definition even where the scheme deviates from ideal conditions, discussing the PDC fidelity separately.

Figure 3 shows the PDC probability as a function of the input frequency $\omega_{\text {in }}$ and the detuning $\Delta=\epsilon_{1}-\omega$. At the two vertical "ridges," $P_{\mathrm{PDC}}$ becomes maximal since at each of these ridges the input frequency $\omega_{\text {in }}$ matches either one of the two frequencies of the vacuum Rabi doublet $|3 \omega\rangle \leftrightarrow|2\rangle$. Therefore, the frequency difference between those ridges 


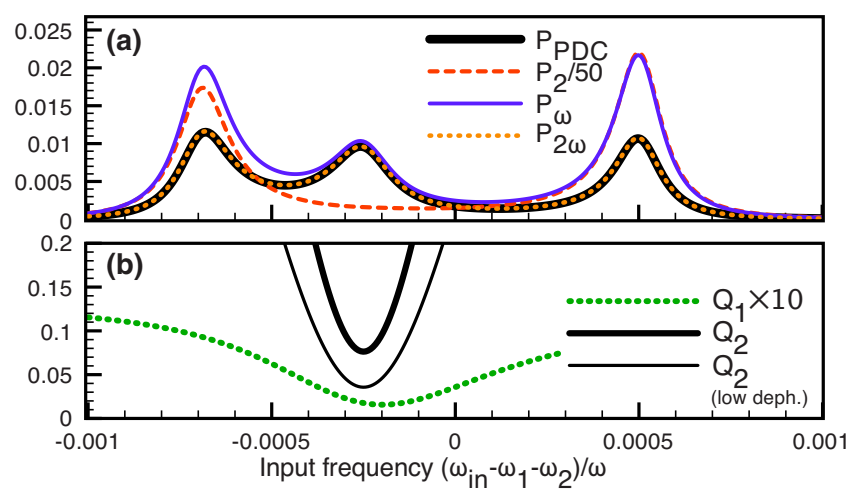

FIG. 4. (Color online) Top: The parametric down-conversion probability $P_{\mathrm{PDC}}$ as a function of input frequency along the cross section indicated in Fig. 3, and the probabilities $P_{\omega}=f P(|\omega\rangle)$ and $P_{2 \omega}=f P(|2 \omega\rangle)$ for one or two photons in the cavity, rescaled in the same manner, with $f=\kappa^{2} /|\alpha|^{2}$ (ideally $P_{\mathrm{PDC}}=P_{\omega}=P_{2 \omega}$ ). The probability of an excited qubit, $P_{2}=f P(|2\rangle)$, is also shown. Bottom: The nonideality parameters $\left.Q_{1}=\gamma P_{|1,2 \omega\rangle} /\left(\kappa P_{\mid \omega, 2 \omega}\right\rangle\right)$ and $Q_{2}$ $=P_{|\omega\rangle} / P_{|\omega, 2 \omega\rangle}-1$, reaching a minimum at the middle peak (thin line: for half the dephasing rate of $\left.\gamma_{\varphi} / \omega=2 \times 10^{-4}\right)$.

corresponds to the vacuum Rabi splitting induced by the coupling $g_{3}$. In addition, we observe that there is a third resonance (dashed curve) at $\omega_{\text {in }}=3 \omega-\widetilde{g}_{1}^{2} / \Delta$. This corresponds to a situation where $\omega_{\text {in }}$ matches the energy of the outgoing state. The energy of that state is shifted dispersively, with the shift depending on the detuning $\Delta$ and the vacuum Rabi frequency $\tilde{g}_{1}=g_{1}\langle 1|\hat{N}| 0\rangle$. Interestingly, therefore, both the dispersive regime and the vacuum Rabi regime occur in this system simultaneously, in contrast to the usual two-level Cooper-pair box coupled to a cavity. We note as well that the result for $P_{\mathrm{PDC}}$ is not symmetric vs $\omega_{\text {in }} \mapsto 3 \omega-\omega_{\text {in }}$ since only $\Delta>0$ is shown here.

However, we note that a maximal PDC probability $P_{\mathrm{PDC}}$ does not guarantee ideal photon pairs, as will be discussed now. The unwanted decay routes, induced by the solid state environment, are (i) $\quad|2\rangle \mapsto|1\rangle \leftrightarrow|\omega\rangle \mapsto|0\rangle$, $|\omega, 2 \omega\rangle \mapsto|\omega\rangle \leftrightarrow|1\rangle \mapsto|0\rangle$, (iii) $|1,2 \omega\rangle \mapsto|1\rangle \mapsto|0\rangle$, and (iv) $|1,2 \omega\rangle \mapsto|2 \omega\rangle \mapsto|0\rangle$. Process (i) leads to a single $\omega$ photon being emitted, while (ii)-(iv) produce a single $2 \omega$ photon with no corresponding partner photon. All of these processes get suppressed with an increasing detuning $|\Delta|=\left|\epsilon_{1}-\omega\right|$, unless this is counteracted by a larger broadening of the levels (produced by dephasing or decay). In order to quantify these processes, we have plotted, in Fig. 4 (bottom), the "nonideality measures" $Q_{1,2}$. Here, $\left.Q_{1}=\gamma P_{|1,2 \omega\rangle} /\left(\kappa P_{\mid \omega, 2 \omega}\right\rangle\right)$ gives the ratio of unwanted relaxation from the intermediate state $|1,2 \omega\rangle$ to the rate of pair emission, while $Q_{2}=P_{|\omega\rangle} / P_{|\omega, 2 \omega\rangle}$ - 1 should vanish in the ideal case when $P_{|\omega\rangle}=P_{|\omega, 2 \omega\rangle}$. The doublet peaks mentioned above yield a large PDC rate, but also a large population of the CPB excited state $|2\rangle$, leading to the decay process (i) and a resulting surplus of $\omega$ photons (Fig. 4, top). Thus, we observe that the vacuum Rabi splitting of the doublet (i.e., the strong-coupling regime) is essential: It allows for the appearance of the third (middle) peak in $P_{\text {PDC }}$ that has a far lower qubit population and corresponding rate of unwanted loss processes (minima in $Q_{1,2}$ ). Any reduction in the broadening of the peaks (set by $\kappa, \gamma, \gamma_{\varphi}$ ) helps to further increase the quality of PDC.

In Fig. 5, we sweep through the parameter space $\left(E_{J}, \delta N_{G}\right)$ or, equivalently, $\left(\epsilon_{1}, \epsilon_{2}\right)$, at each point selecting an input frequency $\omega_{\text {in }}$ which minimizes $Q_{1}$.

The PDC quality is also reflected in the two-photon correlator $K_{j l}(t)=\left\langle\hat{a}_{l}^{\dagger} \hat{a}_{j}^{\dagger}(t) \hat{a}_{j}(t) \hat{a}_{l}\right\rangle /\left(\left\langle\hat{a}_{l}^{\dagger} \hat{a}_{l}\right\rangle\left\langle\hat{a}_{j}^{\dagger} \hat{a}_{j}\right\rangle\right)$, which determines the probability to detect a mode $j$ photon at time $t$ inside the cavity, provided a mode $l$ photon has been detected at $t=0$. Using the quantum regression theorem applied to our master equations, we have checked that the ideal case is approached for small $Q_{1,2}$, where the correlator decays at a rate $\kappa$, both for $(j, l)=(1,2)$ and for $(j, l)=(2,1)$.

We conclude that while maintaining a good reliability of the PDC process, the down-conversion probability can become on the order of a few percent in the present setup, which thus indeed represents a highly efficient source of
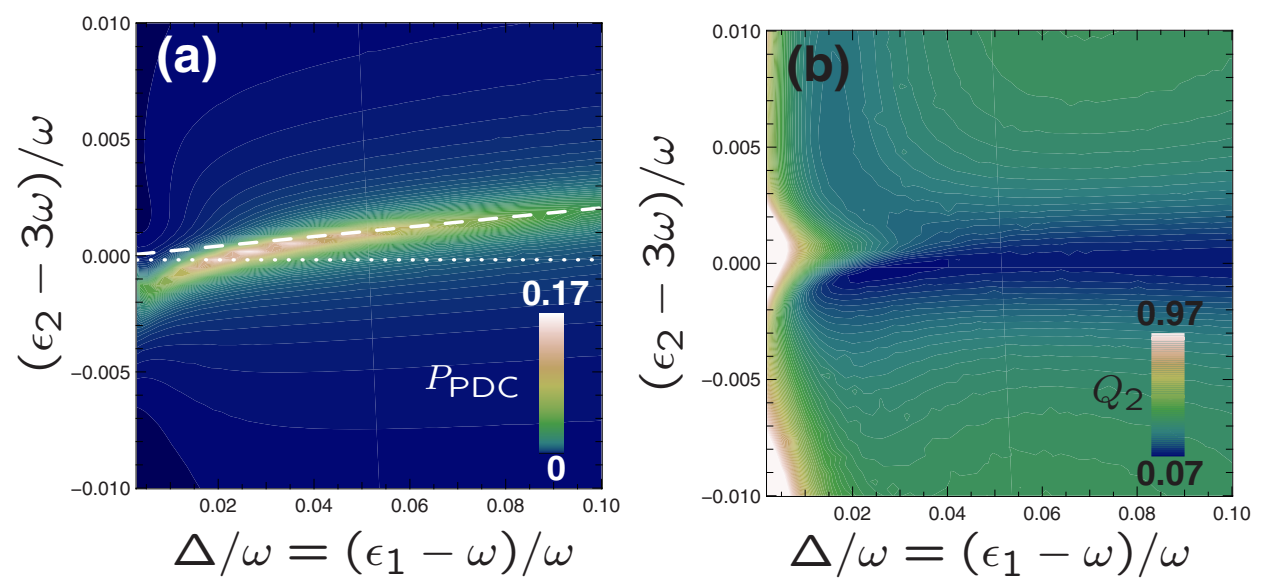

FIG. 5. (Color online) (a) Parametric down-conversion probability $P_{\mathrm{PDC}}$ and (b) the nonideality parameter $Q_{2}$ as a function of the detunings between the Cooper-pair box energy levels $\epsilon_{1,2}$ and the cavity modes (controlled by $E_{J}$ and $N_{G}$ ). At each point, the microwave input frequency $\omega_{\text {in }}$ has been chosen to minimize the parameter $Q_{1}$ (see Fig. 4). The dotted line in (a) indicates the fixed value of $\epsilon_{2}$ for which Fig. 3 has been plotted (where $P_{\mathrm{PDC}}$ was plotted as a function of $\Delta$ and $\omega_{\text {in }}$ ). The values of $P_{\mathrm{PDC}}$ plotted here correspond to those obtained along the full yellow line in Fig. 3, which indicated the locus of minimum $Q_{1}$ (high quality of emitted photon pairs). 


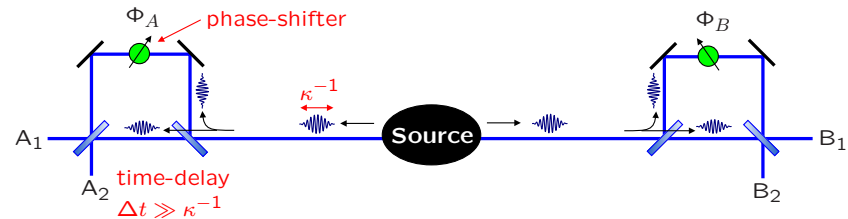

FIG. 6. (Color online) Schematic of the setup needed to generate and test energy-time entanglement according to Franson. A time delay $\Delta t$ between the long and short arms of the Mach-Zehnder interferometers is needed, exceeding the decay time $\kappa^{-1}$ of the cavity producing the photon pairs.

photon pairs. Similar results have been found for other parameter sets [e.g., $g_{0} / \omega=10^{-2}, \quad \kappa_{j} / \omega=10^{-5}, \quad \gamma_{j \leftarrow l} / \omega$ $=10^{-4}(j<l)$, and $\left.\gamma_{\varphi, j} / \omega=10^{-3}(j>0)\right]$.

\section{GENERATION OF ENTANGLEMENT}

The down-converted $\omega$ - and $2 \omega$-photons can independently leak out of either side of the cavity. By postselecting (see Refs. 3 and 25) only events where a photon is detected both in the left and the right arm each, one ends up with a frequency-entangled state that is directly equivalent to the entangled triplet state: $|2 \omega\rangle_{L} \otimes|\omega\rangle_{R}+|\omega\rangle_{L} \otimes|2 \omega\rangle_{R}$. We note, however, that a full Bell test requires measurements in a superposition basis, which is hard to realize for states of different energies.

Another, simpler, possibility is to test for energy-time entanglement, as first proposed by Franson.,7,26 This requires feeding the generated photons into Mach-Zehnder interferometers, each of them containing a short and a long arm as well as a variable phase shifter in one of the arms (see Fig. 6). By measuring the photon-detection correlation between the altogether four output ports of the two interferometers, it is possible to violate the usual kinds of Bell inequalities. The great advantage of such a scheme (particularly in the context of superconducting circuit QED) is that it does not require the polarization as a degree of freedom.

A less demanding, first experimental test of the PDC source described here might measure the intensity cross cor- relations of the microwave output beams (at $\omega$ and $2 \omega$ ) or implement homodyning techniques ${ }^{6,27}$ to characterize the quantum state. Finally, it is worth noting that for $\omega_{3} \approx \epsilon_{2}$, a sufficiently strong vacuum Rabi splitting between $|3 \omega\rangle$ and $|2\rangle$, in principle, enables a scheme where a Rabi $\pi$ pulse is used to put exactly one excitation into the system, which then decays in the way described here, thus realizing a source of microwave photon pairs on demand.

\section{CONCLUSIONS}

In this paper, we have described and analyzed a setup for parametric down-conversion in superconducting circuit cavity QED, suitable for the generation of pairs of entangled microwave photons. In contrast to earlier discussions, we have considered employing a transition via the first three levels of the artificial atom (Cooper-pair box), which can be tuned to achieve a drastically enhanced PDC rate. We have analyzed the tradeoff between optimizing the PDC rate and minimizing loss processes by carrying out extensive numerical simulations of the quantum-dissipative dynamics. The setup described here can be realized by moderate modifications of existing experiments, and it can hopefully form the basis for more detailed investigations into the nonclassical properties of the microwave field in circuit QED experiments.

Note added in proof Recent experiments using the so-called "Transmon" qubit design in the group of R. Schoelkopf at Yale have produced considerably improved charge dephasing times, thus increasing the chances of implementing the present scheme.

\section{ACKNOWLEDGMENTS}

I thank S. Girvin, A. Wallraff, A. Blais, J. Majer, D. Schuster, M. Mariantoni, E. Solano, and R. Schoelkopf for discussions, and especially M. H. Devoret for pointing out the potential use of a three-level configuration. This work was supported by the DFG through SFB 631.
${ }^{1}$ D. C. Burnham and D. L. Weinberg, Phys. Rev. Lett. 25, 84 (1970)

${ }^{2}$ C. K. Hong and L. Mandel, Phys. Rev. Lett. 56, 58 (1986).

${ }^{3}$ Y. H. Shih and C. O. Alley, Phys. Rev. Lett. 61, 2921 (1988).

${ }^{4}$ J. Brendel, E. Mohler, and W. Martienssen, Europhys. Lett. 20, 575 (1992)

${ }^{5}$ P. G. Kwiat, K. Mattle, H. Weinfurter, A. Zeilinger, A. V. Sergienko, and Y. Shih, Phys. Rev. Lett. 75, 4337 (1995).

${ }^{6}$ A. I. Lvovsky, H. Hansen, T. Aichele, O. Benson, J. Mlynek, and S. Schiller, Phys. Rev. Lett. 87, 050402 (2001).

${ }^{7}$ P. R. Tapster, J. G. Rarity, and P. C. M. Owens, Phys. Rev. Lett. 73, 1923 (1994).

${ }^{8}$ C. H. Bennett and D. P. DiVincenzo, Nature (London) 404, 247 (2000).
${ }^{9}$ A. Blais, R. S. Huang, A. Wallraff, S. M. Girvin, and R. J. Schoelkopf, Phys. Rev. A 69, 062320 (2004).

${ }^{10}$ A. Wallraff, D. I. Schuster, A. Blais, L. Frunzio, R. S. Huang, J. Majer, S. Kumar, S. M. Girvin, and R. J. Schoelkopf, Nature (London) 431, 162 (2004).

${ }^{11}$ V. Bouchiat, D. Vion, P. Joyez, D. Esteve, and M. H. Devoret, Phys. Scr., T T76, 165 (1998).

${ }^{12}$ Y. Nakamura, Y. A. Pashkin, and J. S. Tsai, Nature (London) 398, 786 (1999).

${ }^{13}$ J. E. Mooij, T. P. Orlando, L. Levitov, L. Tian, C. H. van der Wal, and S. Lloyd, Science 285, 1036 (1999).

${ }^{14}$ Y. Makhlin, G. Schön, and A. Shnirman, Rev. Mod. Phys. 73, 357 (2001).

${ }^{15}$ I. Chiorescu, P. Bertet, K. Semba, Y. Nakamura, C. J. P. M. Har- 
mans, and J. E. Mooij, Nature (London) 431, 159 (2004).

${ }^{16}$ D. I. Schuster, A. Wallraff, A. Blais, L. Frunzio, R. S. Huang, J. Majer, S. M. Girvin, and R. J. Schoelkopf, Phys. Rev. Lett. 94, 123602 (2005).

${ }^{17}$ A. Wallraff, D. I. Schuster, A. Blais, L. Frunzio, J. Majer, M. H. Devoret, S. M. Girvin, and R. J. Schoelkopf, Phys. Rev. Lett. 95, 060501 (2005).

${ }^{18}$ F. Helmer, M. Mariantoni, A. G. Fowler, J. v. Delft, E. Solano, and F. Marquardt, arXiv:0706.3625 (unpublished).

${ }^{19}$ M. Oberparleiter and H. Weinfurter, Opt. Commun. 183, 133 (2000)

${ }^{20}$ O. Benson, C. Santori, M. Pelton, and Y. Yamamoto, Phys. Rev. Lett. 84, 2513 (2000).
${ }^{21}$ O. Gywat, G. Burkard, and D. Loss, Phys. Rev. B 65, 205329 (2002).

${ }^{22}$ C. Emary, B. Trauzettel, and C. W. J. Beenakker, Phys. Rev. Lett. 95, 127401 (2005).

${ }^{23}$ K. Moon and S. M. Girvin, Phys. Rev. Lett. 95, 140504 (2005).

${ }^{24}$ K. Edamatsu, G. Oohata, R. Shimizu, and T. Itoh, Nature (London) 431, 167 (2004).

${ }^{25}$ A. Aspect, J. Dalibard, and G. Roger, Phys. Rev. Lett. 49, 1804 (1982).

${ }^{26}$ J. D. Franson, Phys. Rev. Lett. 62, 2205 (1989).

${ }^{27}$ M. Mariantoni, M. J. Storcz, F. K. Wilhelm, W. D. Oliver, A. Emmert, A. Marx, R. Gross, H. Christ, and E. Solano, arXiv:cond-mat/0509737 (unpublished). 